\title{
Triangular Silver Nanoparticles: Their Preparation, Functionalisation and Properties
}

\author{
J.M. Kelly ${ }^{a, *}$, G. KeEgAN ${ }^{a}$ And M.E. Brennan-Fournet ${ }^{b}$ \\ ${ }^{a}$ School of Chemistry, University of Dublin, Trinity College, Dublin 2, Ireland \\ ${ }^{b}$ School of Physical Sciences, Dublin City University, Dublin 9, Ireland
}

\begin{abstract}
This paper reports our progress towards developing a reproducible and rapid method to prepare triangular silver nanoplates. The methods are all based on a seed-mediated procedure involving the reduction of silver ions with ascorbic acid in aqueous solution and have variously involved polyvinylalcohol, citrate, and polystyrenesulphonate as modifiers. The triangular silver nanoplate sols have well defined local surface plasmon resonances, which can be tuned throughout the visible and near IR. Aspect ratio (the ratio of edge length to thickness) is shown to be a fundamental parameter determining the triangular silver nanoplate sol properties including, the position of their local surface plasmon resonances maxima and suppression of local surface plasmon resonance retardation effects. The high ensemble sensitivities of the triangular silver nanoplate sol local surface plasmon resonance to changes in the surrounding refractive index within the spectral range appropriate for biosensing is attributed to their high aspect ratio. Silver nanoparticles are more challenging to functionalise than the analogous gold systems, as they are prone to oxidation and are susceptible to degradation by chloride ions. Two methods to stabilise triangular silver nanoplates (treating with thiols and coating with gold) and the formation of gold nanoboxes from the triangular silver nanoplates are also described.
\end{abstract}

PACS: 78.67.Bf, 81.07.Lk

\section{Introduction}

While the properties of gold nanoparticles have been widely exploited over the last few decades, the similar application of silver nanomaterials has been much more limited due to some apparently less favourable features. Thus the local surface plasmon resonance (LSPR) for spherical silver nanoparticles typically occurs about $390 \mathrm{~nm}$, resulting in their pale yellow colour, while that for gold spheres is at about $520 \mathrm{~nm}$, giving them their characteristic strong cherry red colour. Another disadvantage of unprotected silver nanoparticles is that they readily oxidise in the presence of halide ions, so that a strategy has to be developed to prevent this occurring.

In the past decade a number of research teams have demonstrated that anisotropic nanoparticles of silver can be prepared in a controllable fashion and they have shown that these materials have strikingly different optical properties from their spherical counterparts [1-7]. In this article we describe approaches taken (mainly by our own team) to develop a straightforward and rapid method to prepare highly geometrically uniform triangular silver nanoplate (TSNP) sols with strong tunable extinction spectra in the visible and near IR. We demonstrate the versatility of these TSNPs and especially highlight how their high ensemble LSPR sensitivities to changes in the local refractive index give these TSNP sols great potential for biosensing applications. A de-

\footnotetext{
* corresponding author; e-mail: jmkelly@tcd.ie
}

tailed study of the fundamental parameters underpinning these TSNP sol properties has been carried out. Finally we detail one successful approach to their stabilisation and functionalisation which allows them to be used for biosensing and related applications under physiological media conditions.

\section{Development of preparative methods}

Our approach to the production of TSNPs is based on the reduction of silver ions by ascorbic acid catalysed by "seed" silver nanoparticles. In our initial experiments in this area, seeds were prepared by reducing an $\mathrm{AgNO}_{3}$ solution with $\mathrm{NaBH}_{4}$ in the presence of sodium citrate. The resulting yellow solution, which contains small, approximately spherical particles was then used to catalyse the reduction of silver nitrate ions by ascorbic acid in the presence of an aqueous $1 \%(\mathrm{w} / \mathrm{v})$ solution of polyvinylalcohol [8]. It was found that the visible absorption spectrum of the sample showed two bands depending on the ratio of $\mathrm{Ag}^{+}$ions $/ \mathrm{Ag}$ seeds and to the concentration of polyvinylalcohol used. Four samples were studied in detail. Transmission electron microscopy (TEM) showed that each sample contained a mixture of particles, predominantly spheres, triangles (often truncated) and hexagons. The shortest wavelength band (in the $400 \mathrm{~nm}$ region) was identified as originating from spherical particles, while the longer wavelength feature was assigned to be that of the LSPR of the anisotropic particles. These anisotropic particles were shown to greatly enhance the nonlinear saturable absorption properties of the samples 
probed with $6 \mathrm{~ns}, 532 \mathrm{~nm}$ pulses. This is associated with the high polarizability of the LSPR electrons and the associated local $E$-field enhancements, which can lead to strong nonlinear optical susceptibilities $\left(\chi^{3}\right)$. Thus values of $\chi^{3}$ as high as $1.5 \times 10^{-9}$ esu were recorded for samples with LSPR in the region of the excitation wavelength.

Further efforts were made to develop this method, so as to be able to have a procedure, where the nature of the particles, and hence the colour of the sample, could be readily and reproducibly controlled. This was achieved by a similar method to the above in which the reduction of silver ions by ascorbic acid catalysed by citrate-stabilized silver seeds in the presence of a polymer (here polyvinylpyrrolidone - PVP) was carried out in the presence of various concentrations of tri-sodium citrate [9]. It was demonstrated that the colour of the sol could be readily controlled simply by varying the concentration of the citrate salt. Thus at room temperature in the presence of $0.7 \mathrm{mM}$ citrate the sample is dominated by two peaks at $c a .800 \mathrm{~nm}$ and $410 \mathrm{~nm}$, giving the sample its characteristic green colour, while a concentration of $0.17 \mathrm{mM}$ citrate produced a blue sample having a maxima at $c a .600 \mathrm{~nm}$ and $410 \mathrm{~nm}$ and a very low concentration yielded a red colloid with maxima at 510 and $420 \mathrm{~nm}$ (Fig. 1a). TEM measurements showed that two types of particles (namely spheres - absorbing in the $400 \mathrm{~nm}$ region - and nanoplates, whose plasmon band could be readily tuned over the $500-800 \mathrm{~nm}$ range) were present. It is postulated that the citrate ions play a determining role in the formation of the nanoplates by binding to the $\{111\}$ surface of the particles. The presence of both spheres and TSNPs was suggested to be due to the nature of the seeds. Specifically it was suggested that twinned particles are responsible for the formation of the TSNPs. It was also found that while TSNPs were still produced when the concentration of PVP was reduced, both their size and polydispersity were significantly increased. This suggests that the PVP does not play a major role in forming TSNPs but that it does modify their growth and possibly prevents their coalescence.

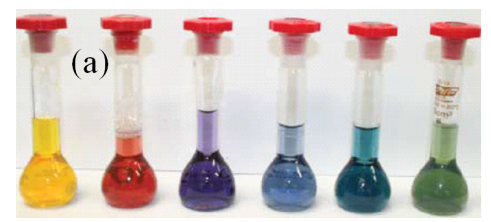

(b)

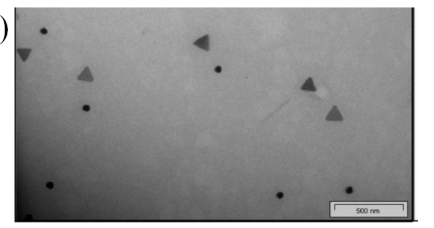

Fig. 1. (a) TSNP sols prepared by the "citrate" method. (b) TEM of samples prepared at $100^{\circ} \mathrm{C}$. Adapted and reproduced by permission from Ref. [9] by permission of the Royal Society of Chemistry.
The reaction was also carried out at higher temperatures. It was found that above $50{ }^{\circ} \mathrm{C}$ the sample contained triangular nanoplates with very well defined shape (as well as small spheres) (Fig. 1b). Interestingly, unlike what was observed at room temperature, where the SPR band shifted to shorter wavelengths within a few hours as the triangular nanoplates became truncated, the samples prepared at higher temperatures were stable in the reaction mixture after preparation. The reason for the stabilisation at higher temperatures is still unexplained. It was also shown that truncation could be induced in the triangular nanoparticles by reaction with a fresh sample of the PVP solution, possibly due to an impurity in the commercial sample used.

While the citrate-controlled procedure did allow for the rapid formation of TSNPS of defined size (and hence colour), the method had the disadvantage that the nanoplates were always accompanied by spheres. As outlined above this was felt to be due to there being at least two types of catalytic seed particles - those that grew into spheres and those which formed the TSNPs. It was therefore decided to try to control the properties of the seeds by adding a polymer to the seed preparation [10]. It was found that the sodium salt of polystyrenesulphonate was particularly effective. (This polymer is closely related to the material used in some water purification ion exchange materials columns.) These polymer-treated seeds are then used to mediate the reduction of the silver nitrate by the ascorbic acid - no other reagent (such as citrate or a polymer) is added at this stage. The samples produced contain essentially only triangular nanoplates, no spheres are formed. After the reaction is complete (a minute or two), citrate is added to stabilise the sample. By varying the ratio of silver seeds to silver ions, the size of the particles can be controlled. This allowed the plasmon band to be tuned so that its maximum could range from about $480 \mathrm{~nm}$ to greater than $1100 \mathrm{~nm}$ (Fig. 2).
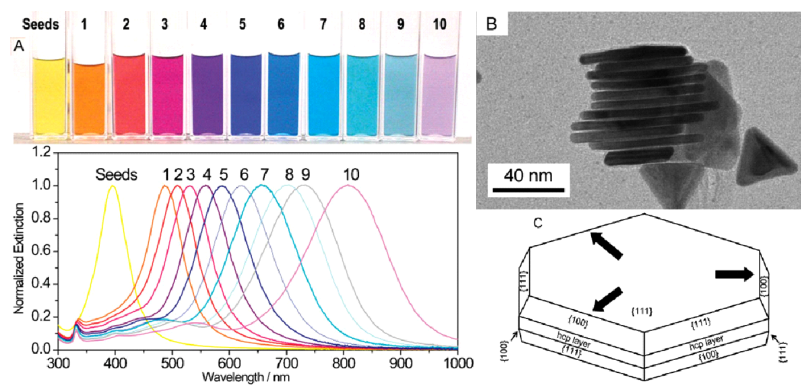

Fig. 2. TSNP sols prepared by the polystyrenesulphonate treated seed particles: (a) samples prepared using various ratios of seeds to $\mathrm{Ag}^{+}$ions, (b) TEM of sample showing particles viewed both from the side and the $\{111\}$ face, (c) proposed structure of growing particles. Adapted and reproduced by permission from Ref. [10].

It is interesting that in this case the growth of the particles is presumably not controlled by absorption on the 
$\{111\}$ face and must be due primarily to the higher reactivity of the atoms on the edges of the nanoplates. Examination of the high resolution TEM images demonstrated the presence of lamellar defects that extend across the crystal, where the silver atoms are arranged in a continuous hexagonal-close-packed (hcp) structure. It is proposed that the growth of the triangular particles is initiated at this hcp layer (Fig. 2c).

This method provides a very easy and rapid method to prepare aqueous suspensions of triangular nanoplates with a particular edge length. It can also be relatively readily scaled up. As outlined above, the growth medium does not contain any polymer, so that the surfaces of the particles should be largely uncontaminated and suitable for functionalisation.

\section{Plasmonic properties of triangular silver nanoplates}

The optical properties of noble metal nanostructures are governed by their unique localized surface plasmon resonance (LSPR). The LSPR consists of the collective oscillation of the metal's conduction electrons along the nanostructure's surface upon excitation by an external resonant electromagnetic field ( $E$-field) [11]. E-field excitation of the LSPR leads to the buildup of polarization charges on the nanostructure surface, which acts as an effective restoring force, allowing the LSPR to occur at a resonant frequency [12]. In silver the inter-band absorption edge is removed from its LSPR, minimizing plasmonic damping which occurs in other metals such as gold and copper for which the LSPR are coincidental with the inter-band transitions. Nanostructure scattering and absorption cross-sections as well as local $E$-field enhancements are strongly enhanced at the LSPR frequency. The LSPR oscillations and their properties are strongly reliant upon factors including size [13, 14], shape [15, 16], dielectric constant $[12,17]$, and the dielectric constant of the surrounding environment $[18,19]$. Here we examine with respect to these factors the LSPR response of the TSNP sols prepared by the polystyrenesulphonate treated seed method outlined above [10].

These TSNP sols exhibit high geometric uniformity, with controllable edge length during synthesis, enabling the systematic tuning of the LSPR throughout the visible and NIR spectrum (Fig. 2a). Size analysis (carried out by TEM and AFM) on these TSNPs highlighted the aspect ratio as a key parameter determining the LSPR properties [19, 20]. A gradual increase in the thickness of the TSNPs was found with increasing edge length for sols with LSPR peak wavelength $\left(\lambda_{\max }\right)$ ranging from $500 \mathrm{~nm}$ to $1150 \mathrm{~nm}$ (Fig. 3a). The aspect ratio of the TSNPs is found to increase from values of 2 to 13 and the ensembles' LSPR $\lambda_{\max }$ is observed to red-shift linearly with increasing aspect ratio (Fig. $3 \mathrm{~b}$ ).

The shape of the polarised electron cloud induced by an incident resonant $E$-field determines the magnitude of the induced restoring force and is an important parameter determining the LSPR and properties such as

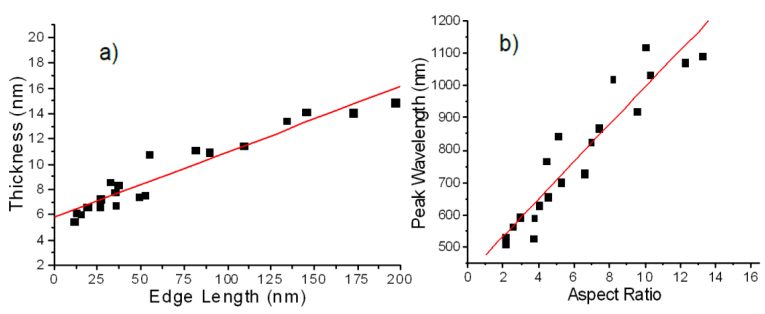

Fig. 3. (a) Linear relationship between the nanoparticle ensembles mean edge length $(\mathrm{nm})$ and mean thickness (nm). (b) TSNP ensembles peak wavelength as a function of mean aspect ratio. Adapted from Ref. [21] and reproduced by permission of the American Chemical Society.

local $E$-field enhancement. Quantitatively, for any general nanostructure shape the dipolar polarizability $(\alpha)$ can be expressed as [20]

$$
\alpha=\frac{\varepsilon_{0} V}{L}\left(\frac{\varepsilon-\varepsilon_{\mathrm{m}}}{\varepsilon+\left(\frac{1-L}{L}\right) \varepsilon_{\mathrm{m}}}\right),
$$

where $L$ is a depolarization factor, which can be related to shape by the shape factor $\chi$ as

$$
\chi=\frac{1-L}{L} \text {. }
$$

The depolarisation factor can be calculated if we consider the TSNPs as oblate spheroid structures with the three axes $A, B$ and $C$ where $A$ (edge length) $=B$ (diagonal) $>C$ (thickness) (Fig. 4a) [21]:

$$
L_{A}=\frac{g(e)}{2 e^{2}}\left[\frac{\pi}{2}-\tan ^{-1} g(e)\right]-\frac{g^{2}(e)}{2}
$$

with

$$
e=\sqrt{1-\left(\frac{B}{A}\right)^{2}}=\sqrt{1-\frac{1}{R^{2}}}
$$

and

$$
g(e)=\left(\frac{1-e^{2}}{e^{2}}\right)^{1 / 2}
$$

where

$$
R=\frac{A}{B}
$$

is the nanostructure aspect ratio.

These equations show that aspect ratio is directly related to the shape factor $\chi$. Figure $4 \mathrm{~b}$ illustrates the calculated shape factor values for the measured TSNPS aspect ratios, which range from 3 up to 18 demonstrating the linear relationship between these two parameters.

Further analysis revealed that aspect ratio plays a primary role in defining and controlling the TSNP LSPR. Preserving the coherence of nanostructure LSPR and maintaining long dephasing time is essential in achieving high local electric field enhancement or high LSPR refractive index sensitivities. The plasmon dephasing time $\left(T_{2}\right)$ determines the homogeneous LSPR spectral linewidth, 


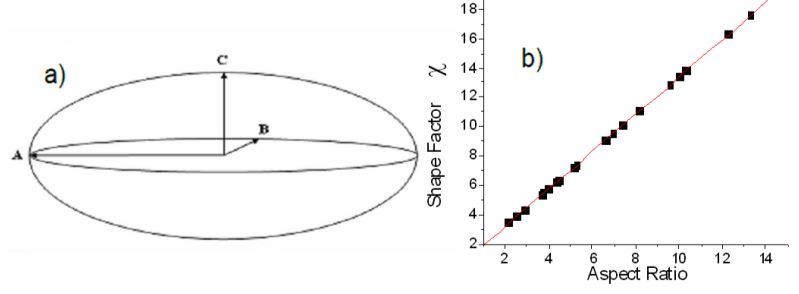

Fig. 4. (a) Oblate ellipsoidal structure with axes $A=$ $B>C$. (b) Linear relationship between the shape factors and aspect ratios of TSNPs.

( $\left.\Gamma_{\text {hom }}\right)$ and is related by $T_{2}=2 \hbar / \Gamma_{\text {hom }}$ thereby identifying narrow linewidths as desirable for optimum LSPR performance. LSPR linewidths are determined by contributions from radiative and nonradiative damping processes and can be expressed as $\Gamma_{\text {hom }}=\Gamma_{\text {rad }}+\Gamma_{\text {nonrad }}$. Interband and intraband (free electron) contributions constitute nonradiative processes. Where the conduction electrons are confined and diffuse on the nanostructure surface, such as in nanostructures with dimensions below the bulk electron mean free path, electron-surface scattering occurs. Taking into account the relative contributions from bulk dephasing, electron-surface scattering and radiation damping, the width of the LSPR $(\Gamma)$ can be described as [22]:

$$
\Gamma=\gamma_{\mathrm{b}}+\frac{A v_{f}}{L_{\mathrm{eff}}}+\frac{\hbar \kappa V}{2},
$$

where $\gamma_{\mathrm{b}}$ is the bulk damping constant $(0.072 \mathrm{eV}$ in silver), $v_{f}$ is the Fermi velocity of electrons in silver, $L_{\mathrm{eff}}$ is the effective mean free path (57 nm in silver), $V$ is the volume and $A$ and $\kappa$ are constants describing the electron surface scattering and volume induced radiation damping contributions, respectively.

Solution phase nanostructures have often been regarded as disadvantageous when compared to single nanostructures and nanostructure arrays due to inhomogeneity within the ensemble resulting in broadened linewidths. The excellent homogeneity with edge length control which we have demonstrated for the TSNP sols prepared from the polystyrenesulfonate sodium salt (PSSS)-coated seeds indicate minimal ensemble averaging effects. From experimental studies on the TSNP sols it was observed that smaller edge length TSNP have broader resonance linewidths than those with larger edge lengths (Fig. 5) [23].

The surface-electron and radiation damping contributions to the evolution of the TSNP sols' linewidths with increasing nanoplate size was examined using the linewidth Eq. (5) [23, 24]. At lower edge lengths, surface electron scattering is found to be prominent. In other nanostructures, the edge length is generally found to increase the contribution from radiative damping processes [24]. However, in the TSNP sols no apparent linewidth increase is observed up to edge lengths of $172 \mathrm{~nm}$. Discrete dipole approximation (DDA) calcula- tions show a similar trend with no increase in the DDA-calculated linewidth values for single large edge length TSNPs (Fig. 5b). Suppression of radiative damping in larger TSNPs occurs, thereby maintaining narrow spectral linewidths. These results also highlight that inhomogeneous broadening effects are minimal and thus enable analysis of the dephasing processes contributing to the linewidth trend for the solution phase TSNP ensembles.

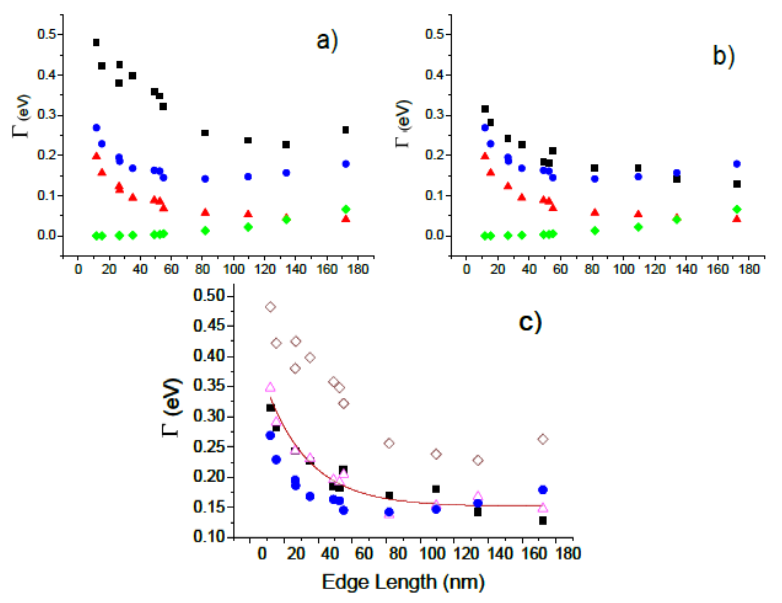

Fig. 5. (a) Linewidth trends recorded for the LSPR with increasing edge lengths of (a) TSNP sols and (b) DDA calculated single TSNP; linewidth (black squares), surface electron scattering (red triangles), radiation damping dephasing (blue circles) and their sum with $A=1$ and $\kappa=0.1 \times 10^{-7} \mathrm{fs}^{-1} \mathrm{~nm}^{-3}$ (green diamonds). (c) Comparison between the fit of the linewidth equation (outlined triangles) to the DDA computed linewidths with $A=1.4$ and $\kappa=1 \times 10^{-7} \mathrm{fs}^{-1} \mathrm{~nm}^{-3}$ (line fit) and the experimentally measured TSNP linewidths (black outlined diamonds). Figure adapted from Ref. [23].

Analysis of the individual absorption and scattering components of DDA-computed extinction spectra highlight aspect ratio as the fundamental parameter demonstrates reduced radiative damping in larger edge length TSNP [23]. Examples of the DDA calculated absorption, scattering and extinction spectra for three TSNPs are shown in Fig. 6. Absorption was observed to be dominant in the smaller TSNPs, accounting for almost $100 \%$ of the extinction spectrum in agreement with theoretical expectations [25]. In nanostructures of larger dimensions, scattering would theoretically be expected to increase to become the dominant process due to the increase in the nanostructure's volume [26]. For TSNP with edge lengths from $55 \mathrm{~nm}$ up to $172 \mathrm{~nm}$ the calculated spectra clearly show that absorption remains the primary process; scattering only becomes significant at edge lengths of $82 \mathrm{~nm}$ and greater.

Confirmation that the dominance of absorption over scattering in the extinction spectra of the TSNP nanostructures is associated with aspect ratio was provided through the analysis of the DDA-computation of TSNPs of similar edge lengths but with varying thicknesses 

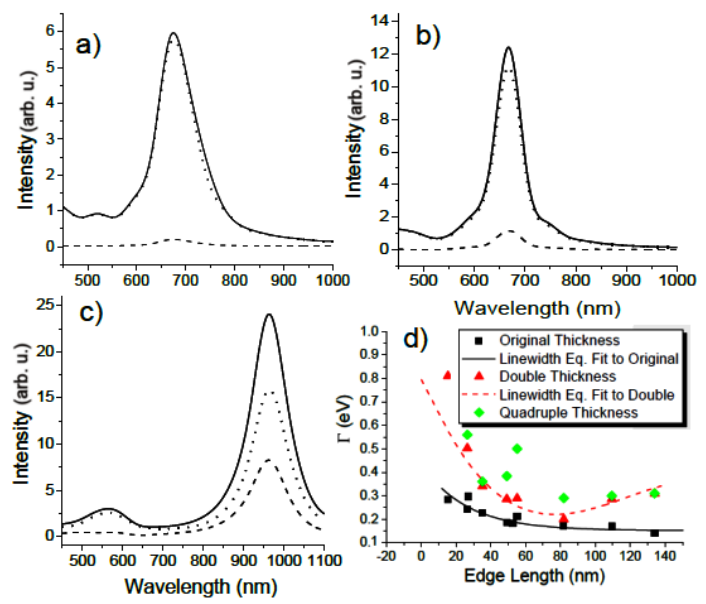

Fig. 6. DDA computed spectra of the extinction (line), absorption (dotted line) and scattering (dashed line) for single TSNP with edge-lengths of (a) $55 \mathrm{~nm}$, (b) $82 \mathrm{~nm}$ and (c) $172 \mathrm{~nm}$. (d) Linewidths of DDA computed spectra for single TSNP nanostructures at the original thickness of the nanoplates, double and quadruple the original thickness. Figure adapted from Ref. [23].

(Fig. 6d). A broadening of the linewidth with increased thickness is clearly observed. The high aspect ratio of the larger edge-length platelet TSNPs acts to reduce retardation effects on the LSPR allowing for the continued coherence of their LSPR.

\section{Biosensing applications}

The LSPR of noble metal nanostructures is a localized surface effect, wherein the LSPR is not confined to the nanostructure surface, but extends into the surrounding dielectric medium (Fig. 7). Changes in the surrounding medium dielectric constant $\varepsilon_{\mathrm{m}}$ or refractive index $n$ influences the LSPR. The extent to which it does will depend upon geometric factors such as size and shape in addition to composition [27]. In nanostructures with LSPRs which are relatively sensitive to changes in the surrounding dielectric, UV-Vis spectroscopy can be used to monitor shifts in the LSPR spectra. Increasing the surrounding refractive index leads to a red-shift in the LSPR (Fig. 7b) while a decrease in refractive index results in a blue shift of the LSPR. The sensitivity of a nanostructure LSPR response to surrounding refractive index changes is defined using a linear refractive index sensitivity $\Delta \lambda / \Delta n\left(\mathrm{~nm} \mathrm{RIU}^{-1}\right)$ [28]. LSPR refractive index sensitivity can be determined as the slope of the plot of the shift observed in the LSPR $\lambda_{\max }$ against the corresponding refractive indices.

TSNPs have a series of key advantages over other nanostructures for biosensing applications. As detailed above, they exhibit versatile LSPR tunability across the visible and into the NIR at wavelengths only limited by the position of water absorption, and silver nanostructures are also advantageous over other noble metal nanoparticles

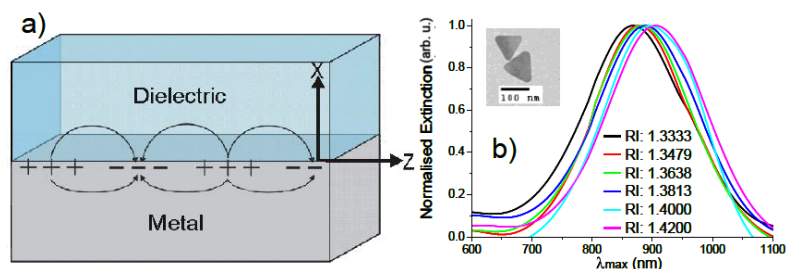

Fig. 7. (a) Plasmon oscillation at the surface of a metal, highlight how the plasmon enters both the metal nanostructure and the surrounding dielectric medium. (b) Shift observed in the LSPR of a TSNP sol resuspended in solutions of varying refractive index. Adapted from Ref [21].

as their LSPR energy is removed from interband transitions resulting in narrower LSPR full width half maxima (FWHM). Within this wavelength range the LSPR of silver nanostructures also exhibit much stronger shifts in response to local dielectric constant changes, than metals such as gold or copper. The highly anisotropic structure and sharp tipped geometries of TSNPs are expected to contribute to increased LSPR sensitivities due to the support of large surface charge polarizability and increased local field enhancement. Significantly if a narrow geometric distribution of the TSNPs within the sols can be achieved, this should lead to a highly uniform response of the ensemble, which can provide statistically relevant data rather than individual readings available using single nanostructure based systems.

In our studies LSPR sensitivities to medium refractive index change were measured using water-sucrose solutions within which the TSNPs were suspended [23]. The spectral shift observed for a $82 \mathrm{~nm}$ edge length TSNP ensemble suspended in the various concentrations of sucrose used is shown in Fig. 7b. TSNP sensitivities are found to increase linearly with LSPR $\lambda_{\max }$ up to $800 \mathrm{~nm}$. A dramatic increase in sensitivity is observed to occur at longer wavelengths with values reaching a maximum of $1096 \mathrm{~nm} \mathrm{RIU}{ }^{-1}$ at a $\lambda_{\max }$ of $1093 \mathrm{~nm}$ for the TSNP sol with an aspect ratio of 13:1 (Fig. 8a).

The highest sensitivities found for these TSNP sols are greater than those reported for various LSPR nanostructures, including those for single nanostructures such as nanorice [29], gold nanorings [30], and gold nanostars [31]. Furthermore unlike other reported high LSPR sensitive nanostructures, the large TSNP sensitivities occur at wavelengths shorter than $1150 \mathrm{~nm}$ (i.e. below the water and biomolecule absorption windows), which can be a significant limiting factor in the suitability of LSPR sensitive nanostructures as biosensors. In addition to this, the TSNP ensemble sensitivity values of $290-433 \mathrm{~nm}$ $\mathrm{RIU}^{-1}$ for sols with LSPR peak wavelengths in the visible exceed those previously reported for nanostructures within this wavelength band such as $205 \mathrm{~nm} \mathrm{RIU}^{-1}$ for single Au triangles by Sherry et al. [32] with LSPR at $631 \mathrm{~nm}$ and $285 \mathrm{~nm} \mathrm{RIU}{ }^{-1}$ for $\mathrm{Au}$ nanorattles in solution with a $\lambda_{\max }$ of approximately $650 \mathrm{~nm}[33]$. In- 


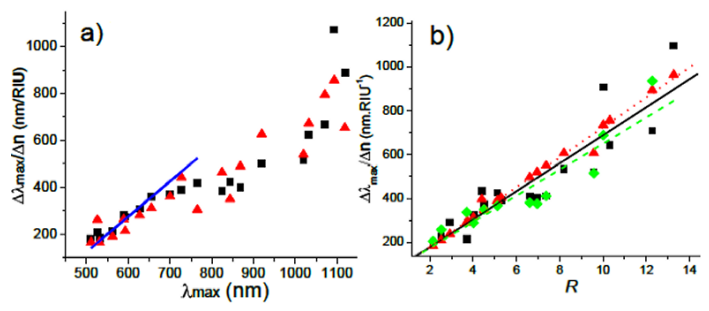

Fig. 8. (a) Experimental sensitivities for TSNPs (black squares), calculated sensitivities (red triangles) and the linear trend predicted by the Miller and Lazarides theory (blue line) with varying LSPR $\lambda_{\max }$. (b) Dependence of the refractive index sensitivity upon aspect ratio experiment (black squares), using the aspect ratio dependent approximation method (red triangles) and discrete dipole approximation calculations (green diamonds). Linear fits for all three methods (experimental fit $=$ black straight line, approximation method $=$ red dotted line, DDA = green dashed line). Calculated sensitivities (red dotted line) show the agreement between the experimental and theoretical values. Adapted and reproduced from Ref. [21] by permission of the American Chemical Society.

terestingly upon comparison to the sensitive response of single LSPR nanostructures it is apparent that minimal ensemble-induced diminution in the LSPR sensitivity values of these TSNP sols is observed.

Miller and Lazarides have developed a model for the maximum sensitivity $\left(\Delta \lambda_{\max } / \Delta n\right)$ of an arbitrary nanostructure LSPR spectrum to the refractive index of the surrounding medium [34]. They predict that the LSPR sensitivity is primarily a function of the spectral location of the nanostructure LSPR and have shown that this holds up to $800 \mathrm{~nm}$. The TSNP values within the 500$800 \mathrm{~nm}$ range are comparable to those optimum values which the Miller theory predicts; however above $800 \mathrm{~nm}$ the trend becomes nonlinear (Fig. 8). We have recently shown that the aspect ratio $(R)$ is directly related to the LSPR sensitivity for the TSNP [23].

DDA calculations of sensitivity values for TSNP with equivalent geometric parameters to those measured experimentally are in agreement with the theory calculated by Miller and Lazarides (Fig. 8b) for wavelengths within the $500 \mathrm{~nm}$ to $800 \mathrm{~nm}$ region, and they further accurately predict the LSPR sensitivities measured experimentally at longer wavelengths in the NIR. This agreement between experimental results and theoretical considerations emphasizes the importance of the aspect ratio and hence shape factor of a nanostructure in determining its LSPR sensitivity to refractive index changes.

These results indicate that these high ensemble local refractive index sensitivity TSNP sols can potentially act as efficient sensors for bioassay applications. They encompass aspect ratios large enough to provide high LSPR sensitivity while the LSPR $\lambda_{\max }$ remains within the spectral range appropriate for biosensing. This represents a promising step toward realizing optimal nanostructures

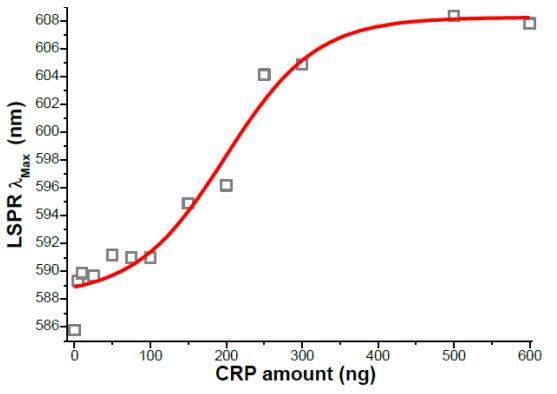

Fig. 9. Dose response curve for the detection of C-reactive protein using phosphocholine functionalised TSNP [35]).

for versatile biosensing. An example of such detection for an acute phase protein, C-reactive protein (CRP) is shown in Fig. 9 [35]). TSNPs functionalised with phosphocholine as a receptor for CRP exhibit a systematic LSPR red shift on the addition of $5 \mathrm{ng}$ to $700 \mathrm{ng}$ of CRP. These results demonstrate the effective translation of the high TSNP LPSR sensitivities into one step assay method for low concentration bioanalyte detection.

\section{Stabilisation and functionalisation}

A disadvantage and challenge with silver nanoparticles is their instability, particularly the ease by which they oxidise. The use of nanoparticles, in future applications, relies on the properties of the particle being maintained over an extended time period, and thus the long-term stability of the particle is a key requirement. Where it is necessary to further functionalise these particles, it is also essential to maintain the particle stability and useful properties of these systems during the functionalisation process. In addition, TSNPs offer an extra challenge to functionalise as the high energy edges of triangular nanoplates are prone to restructuring and some TSNPs have been observed to undergo a morphological change after preparation. Thus TSNPs prepared by lithography have been shown to become thicker and rounded when transferred into solution [36], while those prepared using sodium bis(2-ethylhexyl) sulfosuccinate (NaAOT) have been shown to undergo a morphological change to circular discs within $10 \mathrm{~h}$ [37]. As mentioned above, the TSNPs prepared in the presence of PVP and citrate have also been shown to change shape after preparation, with impurities in the PVP shown to accelerate the process [9]. UV light irradiation and heat are also reported to result in the reconstruction of some TSNPs [38]. Of particular importance for some biosensing and functionalisation applications is that TSNPs have been found to degrade rapidly in the presence of halide anions (Fig. 10a) [39]. Several methods have been utilised to stabilise silver nanoparticles, whilst maintaining their desirable properties. Here we will focus on the work that has been done utilising thiols and gold coating. 


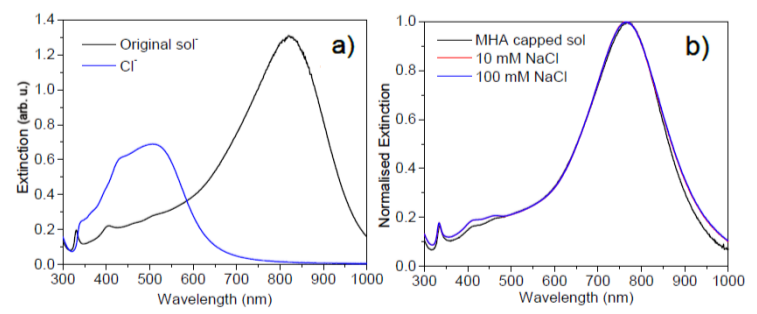

Fig. 10. (a) UV/Vis spectra of TSNPs prepared by method in Ref. [10] before (black) and immediately after the addition of $10 \mathrm{mM} \mathrm{NaCl}$ (blue). (b) UV/Vis spectra of MHA capped TSNPs before (black) and after the addition of 10 (red) and $100 \mathrm{mM} \mathrm{NaCl}$ (blue). Note: TSNPs above are from two separate batches.

Although the silver thiol bond is weaker than the gold thiol bond, there are a number of reports for the successful stabilisation and functionalisation of spherical silver nanoparticles using thiol ligands [40]. The capping of silver particles with neutral ligands, such as the thioalkylated poly(ethylene glycol) described by Doty et al., have been shown to offer excellent stability, but unfortunately this appears to be only applicable to particles with diameters less than $20 \mathrm{~nm}$ [41]. As outlined above, TSNPs are highly susceptible to etching by halide anions so stabilisation is very important. It has been shown that the addition of alkyl thiols (1-hexanethiol, 1-octanethiol, 1-dodecanethiol and 1-hexadecanethiol) to prepared TSNPs "freezes" the morphology of the particles [42], although the nanoplates were only stable if the sodium bis(2-ethylhexyl) sulfosuccinate (NaAOT) was not removed from the solution. $N, N, N$-trimethyl(11-mercaptoundecyl) ammonium bromide has been used to stabilise cetyl trimethylammonium bromide (CTAB) stabilised nanoplates [43]. Multi-dentate thiols have been shown to offer an enhanced stability over monothiols, but this approach may only be applicable to smaller, spherical particles. The monothiols pack more readily on the larger particles due to their reduced curvature [44].

In our group we have used 16-mercaptohexadecanoic acid (MHA) to successfully freeze the morphology of our PSSS-coated seed TSNPs, and we have shown these MHA capped TSNPs to be stable even in $100 \mathrm{mM} \mathrm{NaCl}$ for up to 4 weeks (Fig. 10b) [45]. Shorter alkyl thiols do not show the same long term stability and this contrasting behaviour may be correlated with the packing of the compounds on the surface of the particles. It has been shown that larger shaped particles are more like flat planar structures and the packing is not as high as that of smaller spherical particles [44]. This packing can be correlated with the coverage of the particle. It can be assumed that the advantage and significance of the MHA, is that the long alkyl chain imparts stability to the particles, with the lateral van der Waals interactions creating a well defined and structured self assembled monolayer (SAM). The resulting dense packing creates a hydrophobic area around the particle surface imparting stability to the particles. The significant red shift in the LSPR band upon coating (739 to $772 \mathrm{~nm}$ in the sample used in Fig. 10b) is indicative of formation of a SAM. An advantage of the MHA capping is that not only does it stabilise the particles efficiently against etching, but the introduction of the carboxylic acid group offers us a route to further functionalise these particles.
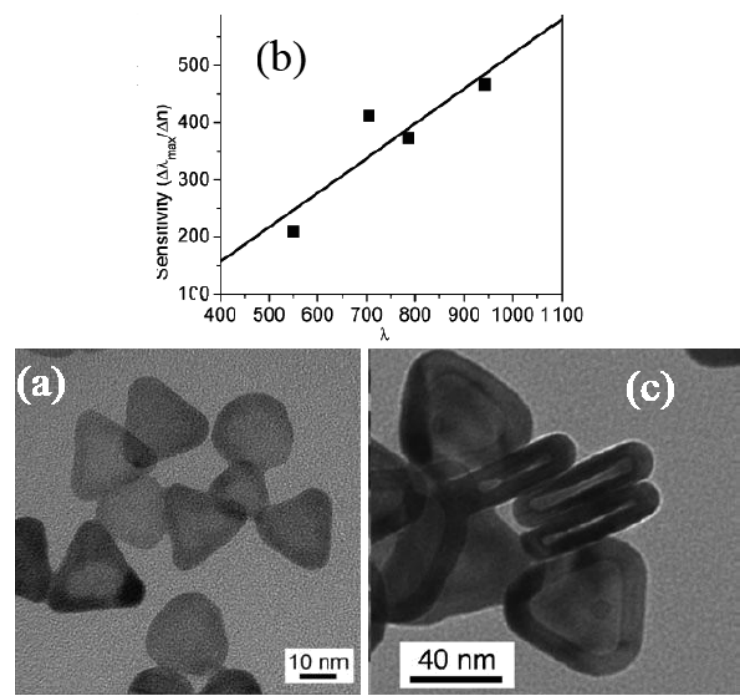

Fig. 11. Gold coated triangular silver nanoprisms prepared by reaction of TSNPs with $\mathrm{HAuCl}_{4}$ (Au:Ag = 0.083:1): (a) TEM images after casting from a $10 \mathrm{mM}$ $\mathrm{NaCl}$ solution. (b) Plot of sensitivity data against the position of the main LSPR. (c) Triangular nanoboxes prepared by reaction of TSNPs with $\mathrm{HAuCl}_{4}(\mathrm{Au}: \mathrm{Ag}=$ 3.3:1). Adapted from Refs. [49] and [50] with permission.

Applying a thin coating of gold to silver nanoparticles might seem to be a simple approach both to stabilisation and functionalisation. However, this can be quite difficult to achieve, because of galvanic replacement. (However, as is noted below, this latter process may give rise to a variety of interesting nanostructures such as nanoshells, nanocages and nanorings [46].) There are a number of reports on the successful gold coating of spherical and triangular silver nanoparticles [47], and gold coating has been shown to sufficiently stabilise spherical silver nanoparticles to allow them to be functionalised with DNA [48]. Aherne et al. have used excess ascorbic acid as a reductant to epitaxially deposit a thin layer of gold on the edge of the PSSS-treated-seed TSNPs [49]. By using a low Au:Ag ratio it was shown that the gold was selectively deposited on the edge of the particles (Fig. 11a). It was found that these particles were stabilised against etching by chloride and it was further concluded that etching by chloride is therefore face selective. The refractive index sensitivity of these particles was also shown to be maintained (Fig. 11b). Indeed the values for the sensitivity of the gold coated TSNPs were actually found to be higher than for the bare TSNPs.

Aherne et al. further extended the above method by 
working with much higher $\mathrm{Au}: \mathrm{Ag}$ ratios thus causing a galvanic replacement reaction. This was shown to cause the formation of triangular hollow AuAg nanoboxes [50], as can be visualised by TEM (Fig. 11c). DDA calculations were also performed to confirm the hollow nature of these particles. Work in progress shows that these materials can be readily functionalised (e.g. by small DNA molecules) and show excellent sensitivity both as medium refractive index probes and for SERRS studies.

\section{Conclusion}

In conclusion routes are now available for the preparation of anisotropic silver nanoparticles, which are both rapid and reproducible and which allow one to exploit the plasmonic behaviour of these nanomaterials. Our work and that of others has shown that TSNPs show great potential for use in a number of applications, not only as biosensors, but also as plasmonic enhancers and as conductive inks.

\section{References}

[1] J.E. Millstone, D.G. Georganopoulou, X. Xu, W. Wei, S. Li, C.A. Mirkin, Small 4, 2176 (2008).

[2] I. Pastoriza-Santos, R.A. Alvarez-Puebla, L.M. Liz-Marzan, Eur. J. Inorg. Chem., 4288 (2010).

[3] I. Pastoriza-Santos, L.M. Liz-Marzan, J. Mater. Chem. 18, 1724 (2008).

[4] A.R Tao, S. Habas, P. Yang, Small 4, 310 (2008).

[5] M. Rycenga, C.M. Cobley, J. Zeng, W. Li, C.H. Moran, Q. Zhang, D. Qin, Y. Xia, Chem Rev. 111, 3669 (2011).

[6] D.M. Ledwith, D. Aherne, J.M. Kelly, in: Metallic Nanomaterials, Ed. C. Kumar, Wiley-VCH, Weinheim 2009, p. 99

[7] D. Aherne, D.M. Ledwith, J.M. Kelly, in: Metal Enhanced Fluorescence, Ed. C.D. Geddes, Wiley, Hoboken 2010 , p. 295.

[8] A.M. Whelan, M.E. Brennan, W.J. Blau, J.M. Kelly, J. Nanosci. Nanotechnol. 4, 66 (2004).

[9] D.M. Ledwith, A.M. Whelan, J.M. Kelly, J. Mater. Chem. 17, 2459 (2007).

[10] D. Aherne, D.M. Ledwith, M. Gara, J.M. Kelly, Adv Funct. Mater. 18, 2005 (2008).

[11] K.A. Willets, R.P. Van Duyne, Ann. Rev. Phys. Chem. 58, 267 (2007).

[12] P.K. Jain, M.A. El-Sayed, Chem. Phys. Lett. 487, 153 (2010).

[13] A. Pinchuk, G. von Plessen, U. Kreibig, J. Phys. D, Appl. Phys. 37, 3133 (2004).

[14] C. Noguez, Opt. Mater. 27, 1204 (2005).

[15] A.J. Haes, R.P. Van Duyne, Anal. Bioanal. Chem. 379, 920 (2004).

[16] B.J. Wiley, S.H. Im, Z.-Y. Li, J. McLellan, A. Siekkinen, Y. Xia, J. Phys. Chem. B 110, 15666 (2006).

[17] C. Voisin, N. Del Fatti, D. Christofilos, F. Vallee, J. Phys. Chem. B 105, 2264 (2001).
[18] T.R. Jensen, M.L. Duval, K.L. Kelly, A.A. Lazarides, G.C. Schatz, R.P. Van Duyne, J. Phys. Chem. B $\mathbf{1 0 3 ,}$ 9846 (1999).

[19] J.J. Mock, D.R. Smith, S. Schultz, Nano Lett. 3, 485 (2003).

[20] C.F. Bohren, D.R. Huffman, Absorption and Scattering of Light by Small Particles, Wiley, New York 1983.

[21] D.E. Charles, D. Aherne, M. Gara, D.M, Ledwith, Y.K. Gun'ko, J.M. Kelly, W.J. Blau, M.E. Brennan-Fournet, ACS Nano. 4, 55 (2010).

[22] E.A. Coronado, G.C. Schatz, J. Chem. Phys. 119, 3926 (2003).

[23] D.E. Charles, M. Gara, D. Aherne, D.M. Ledwith, J.M. Kelly, W.J. Blau, M.E. Brennan-Fournet, Plasmonics 6, 351 (2011).

[24] M. Hu, C. Novo, A. Funston, H. Wang, H. Staleva, S. Zou, P. Mulvaney, Y. Xia, G.V. Hartland, J. Mater. Chem. 18, 1949 (2008).

[25] G. Mie, Ann. Phys. 25, 377 (1908).

[26] S. Link, M.A. El-Sayed, J. Phys. Chem. B 103, 4212 (1999).

[27] H. Chen, X. Kou, Z. Yang, W. Ni, J. Wang, Langmuir 24, 5233 (2008).

[28] L.J. Sherry, R.C. Jin, C.A. Mirkin, G.C. Schatz, R.P. Van Duyne, Nano Lett. 6, 2060 (2006).

[29] H. Wang, D.W. Brandl, F. Le, P. Nordlander, N.J. Halas, Nano Lett. 6, 827 (2006).

[30] E.M. Larsson, J. Alegret, M. Kall, D.S. Sutherland, Nano Lett. 7, 1256 (2007).

[31] C.L. Nehl, H.W. Liao, J.H. Hafner, Nano Lett. 6, 683 (2006).

[32] L.J. Sherry, S.H. Chang, G.C. Schatz, R.P. Van Duyne, B.J. Wiley, Y.N. Xia, Nano Lett. 5, 2034 (2005).

[33] Y. Khalavka, J. Becker, C. Sonnichsen, J. Am. Chem. Soc. 131, 1871 (2009).

[34] M.M. Miller, A.A. Lazarides, J. Phys. Chem. B 109, 21556 (2005).

[35] Y. Zhang, S. Cunningham, P. Fournet, D. Ledwith, M. Voisin, M.E. Brennan-Fournet, unpublished data.

[36] M.D. Malinsky, K.L. Kelly, G.C. Schatz, R.P. Van Duyne, J. Am. Chem. Soc. 123, 1471 (2001).

[37] X. Jiang, Q. Zeng, A. Yu, Nanotechnology 17, 4929 (2006).

[38] Q. Zhang, J. Ge, T. Pham, J. Goebl, Y. Hu, Z. Lu, Y. Yin, Angew. Chem. Int. Ed. 48, 3516 (2009); S. Chen, Z. Fan, D.L. Carroll, J. Phys. Chem. B 106, 10777 (2002); B. Tang, J. An, X. Zheng, S. Xu, D. Li, J. Zhou, B. Zhao, W. Xu, J. Phys. Chem. C 112, 18361 (2008). 
[39] X.C. Jiang, A.B. Yu, Langmuir 24, 4300 (2008); J. An, B. Tang, X. Zheng, J. Zhou, F. Dong, S. Xu, Y. Wang, B. Zhao, W. Xu, J. Phys. Chem. C 112, 15176 (2008); S.-H. Ciou, Y.-W. Cao, H.-C. Huang, D.-Y. Su, C.-L. Huang, J. Phys. Chem. C 113, 9520 (2009); S. Xu, B. Tang, X. Zheng, J. Zhou, J. An, X. Ning, W. Xu, Nanotechnology, 20, 415601 (2009); B. Tang, S. Xu, J. An, B. Zhao, W. Xu, J.R. Lombardi, Phys. Chem. Chem. Phys. 11, 10286 (2009); M.-S. Hsu, Y.-W. Cao, H.-W. Wang, Y.-S. Pan, B.-H. Lee, C.-L. Huang, Chem. Phys. Chem. 11, 1742 (2010); P. Yu, J. Huang, C.-T. Yuan, J. Tang, J. Chin. Chem. Soc. 57, 528 (2010).

[40] S.A. Harfenist, Z.L. Wang, M.M. Alvarez, I. Vezmar, R.L. Whetten, J. Phys. Chem. 100, 13904 (1996); C.P. Collier, R.J. Saykally, J.J. Shiang, S.E. Henrichs, J.R. Heath, Science 277, 1978 (1997); B.A. Korgel, S. Fullam, S. Connolly, D. Fitzmaurice, J. Phys. Chem. B 102, 8379 (1998); D.G. Thompson, A. Enright, K. Faulds, W.E. Smith, D. Graham, Anal. Chem. 80, 2805 (2008).

[41] R.C. Doty, T.R. Tshikhudo, M. Brust, D.G. Fernig, Chem. Mater. 17, 4630 (2005).

[42] X. Jiang, Q. Zeng, A. Yu, Langmuir 23, 2218 (2007).

[43] X. Le Guevel, F.Y. Wang, O. Stranik, R. Nooney, V. Gubala, C. McDonagh, B.D. MacCraith, J. Phys. Chem. C 113, 16380 (2009).

[44] B.C. Mei, E. Oh, K. Susumu, D. Farrell, T.J. Mountziaris, H. Mattoussi, Langmuir 25, 10604 (2009).
[45] G. Keegan, Ph.D. Thesis, University of Dublin, Trinity College, Dublin, 2011.

[46] B.G. Prevo, S.A. Esakoff, A. Mikhailovsky, J.A. Zasadzinski, Small 4, 1183 (2008); J. Chen, J.M. McLellan, A. Siekkinen, Y. Xiong, Z.-Y. Li, Y. Xia, J. Am Chem. Soc. 128, 14776 (2006); G.S. Metraux, Y.C. Cao, R. Jin, C.A. Mirkin, Nano Lett. 3, 519 (2003); Y. Sun, Y. Xia, Adv. Mater. 15, 695 (2003); L.-P. Jiang, S. Xu, J.-M. Zhu, J.-R. Zhang, J.-J. Zhu, H.-Y. Chen, Inorg. Chem. 43, 5877 (2004).

[47] Y.W. Cao, R. Jin, C.A. Mirkin, J. Am. Chem. Soc. 123, 7961 (2001); S. Pande, S.K. Ghosh, S. Praharaj, S. Panigrahi, S. Basu, S. Jana, A. Pal, T. Tsukuda, T. Pal, J. Phys. Chem. C 111, 10806 (2007); R.G. Sanedrin, D.G. Georganopoulou, S. Park, C.A. Mirkin, Adv. Mater. 17, 1027 (2005); B. Rodríguez-González, A. Burrows, M. Watanabe, C.J. Kiely, L. Liz-Marzan, J. Mater. Chem. 15, 1755 (2005).

[48] Y. Cao, R. Jin, C.A. Mirkin, J. Am. Chem. Soc. 123 7961 (2001).

[49] D. Aherne, D.E. Charles, M.E. Brennan-Fournet, J.M. Kelly, Y.K. Gun'ko, Langmuir 25, 10165 (2009).

[50] D. Aherne, M. Gara, J.M. Kelly, Y.K. Gun'ko, Adv. Funct. Mater. 20, 1329 (2010). 